\title{
Acute-onset Paraplegia in Post-operative D3-D5 Meningioma Excision due to Intraspinal Oxidized Cellulose
}

\author{
Mohit M. Kukreja, Vidyadhar G. Telang \\ Department of Orthopaedics, Dhanvantari Orthopaedic Hospital \& Research Centre, Mumbai, India.
}

\begin{abstract}
Corresponding Author:
Dr. Mohit M.Kukreja

Email:drmohitkukreja@gmail.com

This is an Open Access article distributed under the terms of the Creative Commons Attribution License (creativecommons.org/ licenses/by/3.0).
\end{abstract}

Received : March 29, 2017

Accepted : June 6, 2017

Published : July 10, 2017

\begin{abstract}
Background: Intra-spinal oxidized cellulose-induced post-operative paraplegia has been a rare but devastating complication and has been variedly reported. This is one of the common hemostatic agent used and invariably left in-situ. However, oxidized cellulose is associated with the peculiar chemical property to swell and cause pocket-compression, especially in confined areas, which may recover but can also cause an everlasting complete/ partial neurological deficit. Case Report: Our patient, a 68 year old female operated for a D3-D5 level meningioma developed post-surgical acute-onset paraplegia. An immediate post-operative MRI, urgent re-exploration and meticulous decompression resulted in complete neurological recovery at six months post-operatively. Conclusion: We, thus, present this unusual but well-documented oxidized cellulose related complication and make an attempt to highlight and suggest some basic teaching points.
\end{abstract}

Keywords: Humans, Meningioma, Meningeal Neoplasms, Oxidized Cellulose, Paraplegia.

\section{Introduction}

Iatrogenic paraplegia post-thoracic surgery is a rarity but when encountered, the sequelae and the deficit can be devastating for the patient. There have been reports of paraplegia in adults after surgery on the thoracic spine, aorta (thoracic/abdominal) and the chest cavity. Ischemia owing to vascular damage to the intercostal arteries and compression due to epidural hematomas are the two main causes $[1,2]$. However, oxidized cellulose has been implicated as a rare cause also.

\section{Surgicel (oxidized cellulose) original} absorbable hemostat (Johnson \& Johnson Wound Management, a Division ofEthicon, Inc.Somerville, NJ), was approved for use in the United States in 1960 [3]. Since then, it is widely used for intraoperative hemostasis in neurosurgery, thoracic surgery and orthopedics. Oxidized cellulose is thrombogenic material used to control bleeding originating from delicate and/or friable tissues
[4]. It is commercially available as gauze like material and is generally inert and bio-absorbable [4]. The hemostatic action of oxidized cellulose is by formation of a gelatinous mass upon saturation with blood, which leads to formation of a stable clot $[4,5]$. Along with the hemostasis, the oxidized cellulose mass expands and swells due to intrinsic chemical property. This property is particularly significant in a confined space containing neural tissue.

Since the time this specific oxidized cellulose has been in vogue, here have been several reported cases of post-operative paraplegia. Only few cases of paraplegia following the use of oxidized cellulose in thoracic surgery had been reported till 1999 [2,6-9]. We present to you a case report of a post-operative iatrogenic acute-onset complete paraplegia due to oxidized cellulose which was completely reversed by a timely re-exploration and thus, no resultant long-term sequelae. 


\section{Case Report}

Our patient, 68 year old female came with complaints of low back pain (score of 6 out of 10 on visual analog scale) and bilateral limb weakness since last four months. The back pain improved by lying in supine position and was exacerbated on exertion. There was no preceding history of trauma, fall, fever, loss of weight or appetite, bladder or bowel complaints. Physical examination showed local tenderness to palpation and percussion. On examination, there was bilateral lower limb weakness with power $4 / 5$ in all muscle groups. Sensation was normal and there was hyper-reflexia $(3+)$ of the knee and the ankle jerks bilaterally. Fig.1 and Fig.2 show the MRI images of the lesion with the differential given as a neuroma/ meningioma.

The surgery was electively planned for the patient and the lesion excised. The canal was decompressed by doing a liberal laminectomy spanning D3-D5 vertebra. We administered the usual intravenous bolus dose of steroid intraoperatively as there was cord handling and did neuro-monitoring to be sure of no iatrogenic cord damage. The dura was sutured by 5-0 Prolene meticulously. At surgery, there was substantial bleeding that extended through the inter-vertebral area and the spinal canal and hemostasis was achieved by packing with oxidized cellulose and a drain was placed as the usual protocol for these invasive spine surgeries.

Post-operatively, immediately after surgery, power improved to $5 / 5$ in both lower limbs, however, the patient complained of bilateral lower limb paraesthesias with pain in both lower limbs. Gradually, by about 8 hours post-operatively there was flaccid acute paraparesis which progressed to complete paraplegia of both lower limbs with bowel/ bladder involvement and no sensation below the D4 dermatome. We immediately followed it up with a repeat MRI scan on day one to know the cause of this sudden complete

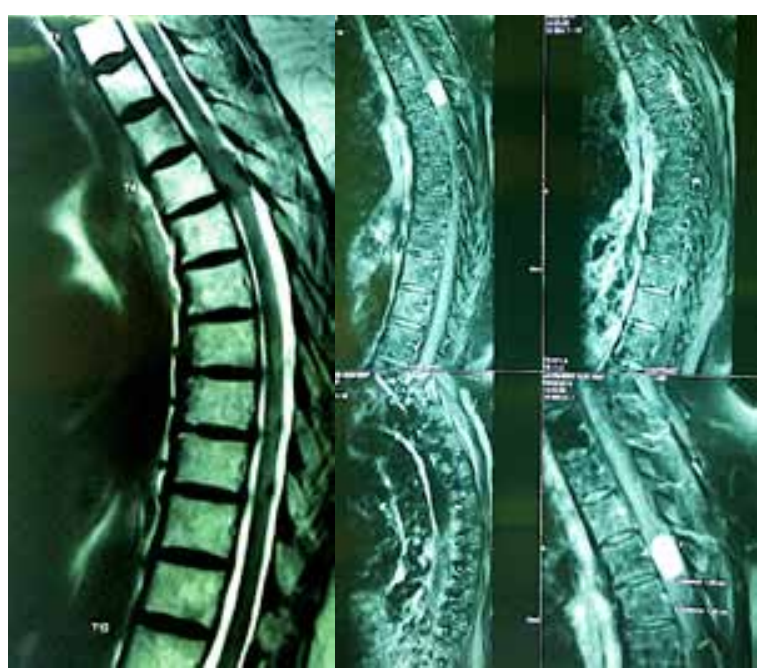

Fig.1: $M R I D-L$ spine, sagittal cuts $T 1 W$ and $T 2 W$ images shows a homogenous well-defined extra-medullary, intradural lesion at the D4 vertebral level.

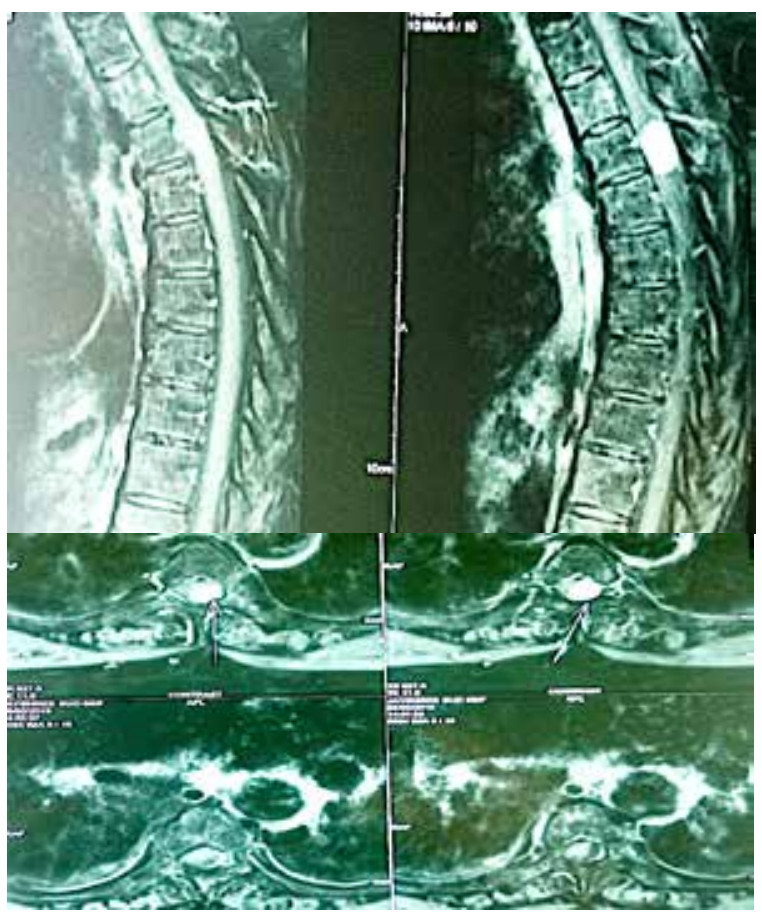

Fig.2: $M R I D-L$ spine with the enlarged $T 2 W$ sagittal cuts and $T 2 W$ axial cuts shows the well-defined mass of about $18 \mathrm{~mm}$ in vertical length and $10 \times 9 \mathrm{~mm}$ in the vertical dimension (extra-medullary, intra-dural).

neurological deterioration post-operatively after having documented no neuro-deficit immediately after surgery. The MRI T2W sagittal and coronal 
cuts are shown in Fig.3. The images indicated a mass that was pushing the spinal cord anteriorly and towards the right. A diagnosis of an extradural hematoma was made.

We re-explored the patient on Day two and the expanded oxidized cellulose with hematoma was removed. Fig.4 above describes the final intraoperative photograph after oxidized cellulose removal. We also reconfirmed adequate decompression, ruled out a dural leak/ tear and achieved wound closure. Histopathology report confirmed the primary lesion as a meningioma. Postoperatively, on day-27, patient regained her bowel/bladder continence and by eight weeks postoperatively, power in lower limbs improved to $4 / 5$. The patient continued intensive physiotherapy and by six months post-surgery (from the first surgery), power in bilateral lower limbs improved to $5 / 5$. At one year follow-up after the first operation, patient has fully recovered with no remnant neurodeficit/ disability.

\section{Discussion}

Hemostasis during surgery benefits the patient, the surgical team and the health care facility [6]. The choice of proper hemostatic agent and the time of its application requires understanding of the mechanism of action, efficacy and possible adverse events of various hemostatic agents [7] including absorbable hemostatic agents, biologically active topical hemostatic agents and systemically administered agents [8].

Bone wax and oxidized cellulose are among the commonly used hemostatic agents for controlling hemorrhage from the surgical site. Inspite of the development of newer materials for hemostasis, the more commonly used materials like oxidized cellulose have not changed in last 50 years. Furthermore, the use of hemostatic agents is not free from health risks and complications can arise as a result of physical attributes of the hemostatic material like mechanical compression or due to phlogistic effects secondary to their chemical nature [5,9-11].

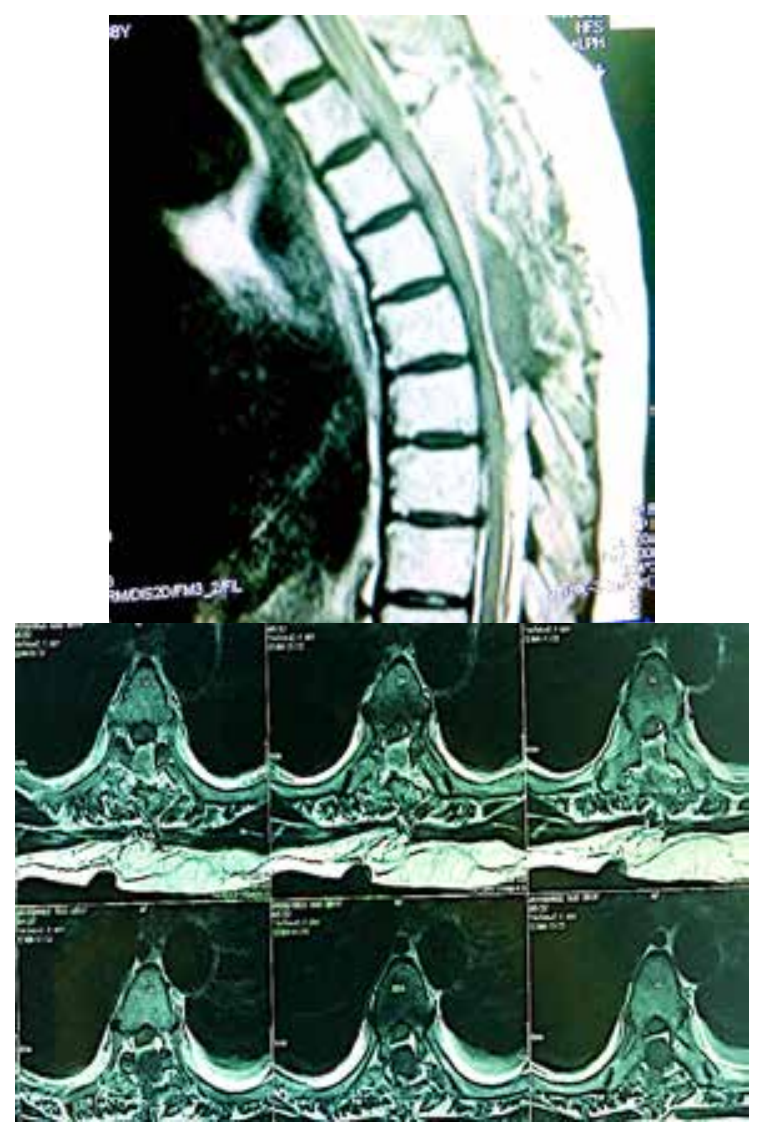

Fig.3: Post-operative MRI scan (coronal and sagittal cuts) showing a hypo-intense well-defined extra-dural, extramedullary mass/collection spanning the D4-D5 region compressing on the cord and causing spinal canal stenosis at that level.

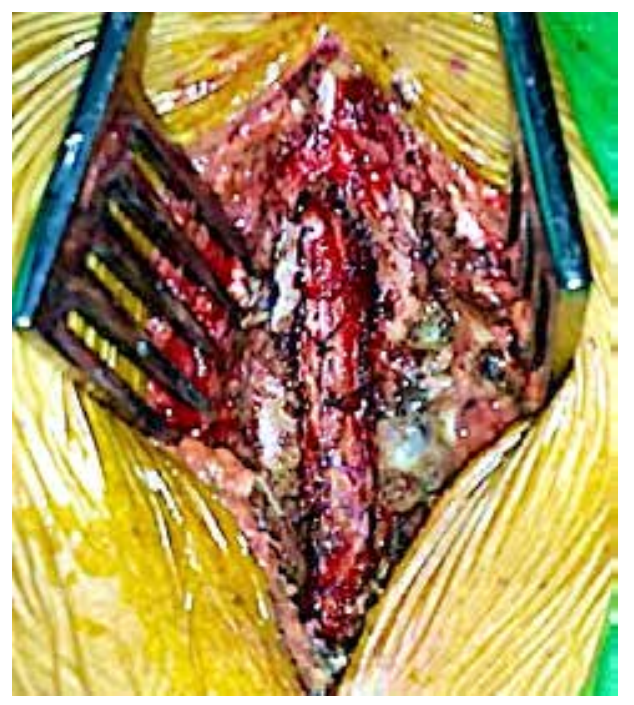

Fig.4: Previously sutured decompressed duramater at the D3-D5 level after removal of the oxidized cellulose and thorough wash. 
Oxidized cellulose is obtained by dissolving alpha cellulose in an alkaline organic solvent [4]. It is usually placed in the surgical site to achieve hemostasis by formation of a gelatinous mass saturated with blood. Advantages of oxidized cellulose as a hemostatic agent include its inertness, bio-absorbability, no foreign body reaction and in-vitro antibacterial effects [4,5]. Nevertheless literature suggests that oxidized cellulose can swell and increase in volume following saturation with blood thereby leading to mechanical compression on vital anatomical structures and radiographic artifacts mimicking abscesses and tumors in CT and ultrasonography [4,12-14].

Oxidized regenerated cellulose is flexible, sheer weave fabric prepared by the controlled oxidation of regenerated cellulose [15]. Because it is flexible, it conforms to irregular surfaces, making it ideal for use during a wide variety of surgical procedures. The sheer weave also allows visualization of the bleeding site. It can be cut or folded to fit the bleeding site and can be removed with gentle irrigation after hemostasis is achieved. The mechanisms by which oxidized regenerated cellulose facilitate hemostasis are not completely understood; however, it is likely that activation of the intrinsic coagulation pathway plays a role [16]. Additional proposed mechanisms include the formation of a gellike layer (matrix) that holds clots in place and vasoconstriction triggered by the low $\mathrm{pH}$ of the product $[16,17]$.

Adverse reactions reported with oxidized cellulose include granulomatous foreign body reactions that mimic brain tumor recurrence; hematoma, paralysis and nerve damage [29-33]. Sassan et al. [23] recommended that oxidized cellulose be removed whenever possible after hemostasis is achieved. This is particularly true when the product is applied in confined areas [2124]. In fact, even the manufacturers recommend its removal following use for laminectomy, arthroplasty and from neural pathways. But still, even today, it is frequently left in situ. Studies have indicated that the extent of damage is timedependent and the delayed evacuation contributes to the lack of neurological recovery $[2,7]$.

Although generally safe and well tolerated, these hemostatic agents should be removed when used around, in or in proximity to foramina in bone, areas of bony confine, the spinal cord, and/or the optic nerve or chiasma because it may otherwise swell and cause unwanted pressure.

\section{Conclusion}

Serious neurological complications may be associated with the use of oxidized cellulose, due to its ability to swell and migrate. Clinical suspicion of this complication is extremely vital for a timely re-exploration and removal of the swollen bloodladen oxidized cellulose. Excess oxidized cellulose should be removed once adequate hemostasis is obtained. Not only the surgeon but every radiologist should also be well aware of this potential cause of acute/delayed-onset paraplegia in a post-operative patient.

Contributors: MMK: manuscript writing, literature search and case management; VGT: manuscript editing, case management and literature search. MMK will act as guarantor. All authors approved the final version of the manuscript.

Funding: None; Competing interests: None stated.

\section{References}

1. Attar S, Hankins JR, Turney SZ, Krasna MJ, McLaughlin JS. Paraplegia after thoracotomy: report of five cases and review of the literature. Ann Thorac Surg. 1995;59:14101416.

2. Wada E, Yonenobu K, Ebara S, Kuwahara O, Ono K. Epidural migration of hemostatic agents as a cause of postthoracotomy paraplegia. J Neurosurg. 1993;78:658660.

3. Levy ML, Day JD, Fukushima T, Batjer HH, Gamache FW Jr. Surgicel Fibrillar absorbable oxidized regenerated cellulose. Neurosurgery. 1997;41:701-702

4. Oto A, Remer EM, O'Malley CM, Tkach JA, Gill IS. MR characteristics of oxidized cellulose (Surgicel). AJR Am J Roentgenol. 1999;172:14814.

5. Dogan S, Kocaeli H, Doygun M. Oxidised regenerated cellulose as a cause of paraplegia after thoracotomy: 
Case report and review of the literature. Spinal Cord. 2005;43:4457.

6. Samudrala S. Topical hemostatic agents in surgery: A surgeon's perspective. AORN J. 2008;88:S211.

7. Achneck HE, Sileshi B, Jamiolkowski RM, Albala DM, Shapiro ML, Lawson JH. A comprehensive review of topical hemostatic agents: Efficacy and recommendations for use. Ann Surg. 2010;251:21728.

8. Lawson JH. The clinical use and immunologic impact of thrombin in surgery. Semin Thromb Hemost 2006;32 Suppl 1:98110.

9. Katre C, Triantafyllou A, Shaw RJ, Brown JS. Inferior alveolar nerve damage caused by bone wax in third molar surgery. Int J Oral Maxillofac Surg. 2010;39:5113.

10. Finn MD, Schow SR, Schneiderman ED. Osseous regeneration in the presence of four common hemostatic agents. J Oral Maxillofac Surg. 1992;50:60812.

11. Schonauer C, Tessitore E, Barbagallo G, Albanese V, Moraci A. The use of local agents: Bone wax, gelatin, collagen, oxidized cellulose. Eur Spine J. 2004;13 Suppl 1:S8996.

12. Alves Júnior L, Vicente WV, Ferreira CA, Manso PH, Arantes LR, Pinheiro KS, et al. Surgicel packing and an erroneous diagnosis of mediastinitis in a neonate. Tex Heart Inst J. 2010;37:1168.

13. Azmy AF. Oxidized cellulose haemostat mimicking a possible recurrence of neuroblastoma. BJU Int. 2001;88:2956.

14. Gao HW, Lin CK, Yu CP, Yu MS, Chen A. Oxidized cellulose (Surgicel) granuloma mimicking a primary ovarian tumor. Int J Gynecol Pathol. 2002;21:4223.

15. Amar A, Levy ML. Applications of topical hemostatic agents in neurosurgery. Contemp Surg Residents. 1996;4:19-22.
16. Krizová P, Másová L, Suttnar J, Salaj P, Dyr JE, Homola $\mathrm{J}$, et al. The influence of intrinsic coagulation pathway on blood platelets activation by oxidized cellulose. J Biomed Mater Res A. 2007;82:274-280.

17. Hong YM, Loughlin KR. The use of hemostatic agents and sealants in urology. J Urol. 2006;176:2367-2374.

18. Sandhu GS, Elexpuru Camiruaga JA, Buckley S. Oxidized cellulose (Surgicel) granulomata mimicking tumour recurrence. Br J Neurosurg. 1996;10:617-619.

19. Ito H, Onishi H, Shoin K, Nagatani H. Granuloma caused by oxidized cellulose following craniotomy. Acta Neurochir Wien. 1989;100:70-73.

20. Kothbauer KF, Jallo GI, Siffert J, Jimenez E, Allen JC, Epstein FJ. Foreign body reaction to hemostatic materials mimicking recurrent brain tumor. Report of three cases. J Neurosurg. 2001;95:503-506.

21. Henry MC, Tashjian DB, Kasowski H, Duncan C, Moss RL. Postoperative paraplegia secondary to the use of oxidized cellulose (Surgicel). J Pediatr Surg. 2005;40:E9E11.

22. Iwabuchi S, Koike K, Okabe T, Tago S, Murakami T. Iatrogenic paraplegia caused by Surgicel used for hemostasis during a thoracotomy: report of a case. Surg Today. 1997;27:969-970.

23. Keshavarzi S, MacDougall M, Lulic D, Kasasbeh A, Levy M. Clinical experience with the surgicel family of absorbable hemostats (oxidized regenerated cellulose) in neurosurgical applications: A Review Wounds. 2013;25:160-167.

24. Menovsky T, Plazier M, Rasschaert R, Maas AI, Parizel PM, Verbeke S. Massive swelling of Surgicel ${ }^{\circledR}$ Fibrillar $^{\mathrm{TM}}$ hemostat after spinal surgery. Case report and a review of the literature. Minim Invasive Neurosurg. 2011;54:257259. 